\title{
MULTIFRACTAL ANALYSIS AND THE VARIANCE OF GIBBS MEASURES
}

\author{
T. Jordan And M. Pollicott \\ Warwick University
}

\begin{abstract}
The multifractal decomposition of Gibbs measures for conformal iterated function system is well known. We look at a finer decomposition which also takes into account the rate of convergence. This is motivated by work by Olsen in the self-similar case. Our study of this finer decomposition involves investigation of the variance of Gibbs measures. This is a problem of independent interest.
\end{abstract}

\section{INTRODUCTION}

Hausdorff dimension is one of the most useful and effective tools in understanding the nature of fractal sets. For example, given a Cantor set $X$ in the real line we can describe its "size" in terms of its Hausdorff dimension [5]. Often we want to consider subsets defined in terms of measures. Let us denote by $B(x, r)=\{y \in$ $X: d(x, y)<r\}$ a ball of radius $r>0$ about a point $x \in X$ then given a reference probability measure $\nu$ on a set $X$ and we can associate its pointwise dimension at $x$ by

$$
d_{\nu}(x)=\lim _{r \rightarrow 0} \frac{\log \nu(B(x, r))}{\log r},
$$

when it exists. Multifractal analysis describes the dimension of the sets of points $x$ for which the limit takes a given value [6], [13], [18]. A particularly successful theory can be developed in the context of dynamically defined sets. Let us consider a dynamically defined Cantor set $X$ supporting a suitable probability measure $\nu$. More precisely, let $X$ be the limit set for a $C^{2}$ iterated function scheme satisfying the strong separation condition and let $\nu$ be a self-similar measure $\nu$, with respect to a Hölder potential $g: X \rightarrow \mathbb{R}$. Let $T: X \rightarrow X$ be the associated expanding map. The multifractal spectrum of $\nu$ describes the set of points whose (symbolic) pointwise dimension function takes different values. We can first decompose the limit set $X$ as

$$
X=\bigcup_{\alpha \in \mathbb{R}} X_{\alpha} \cup X_{\infty}
$$

where $X_{\alpha}=\left\{x: d_{\nu}(x)=\alpha\right\}$, for $\alpha \in \mathbb{R}$, and $X_{\infty}$ denotes the points for which the limit $d_{\nu}(x)$ doesn't exist. The usual (symbolic) multifractal spectrum of the measure $\nu$ describes the Hausdorff dimension $\mathcal{F}(\alpha)=\operatorname{dim}_{H}\left(X_{\alpha}\right)$ of these sets. This important function has been extensively studied by several authors (e.g., [14], 
$[9],[3],[11],[16])$. In this paper we want to consider a finer structure of these sets. More precisely, we define, for each $\gamma \in \mathbb{R}^{+}$,

$$
X_{\alpha, \gamma}=\left\{x: \limsup _{n \rightarrow+\infty} \frac{\log \nu\left(B\left(x,\left|\left(T^{n}\right)^{\prime}(x)\right|^{-1}\right)-\alpha \log \left|\left(T^{n}\right)^{\prime}(x)\right|^{-1}\right.}{\sqrt{n \log \log n}}=\gamma\right\}
$$

We can then write

$$
X_{\alpha}=\bigcup_{\gamma \in \mathbb{R}^{+}} X_{\alpha, \gamma} \cup X_{\alpha, \infty}
$$

where $X_{\alpha, \infty}$ is the set of point such that the limit supremum isn't finite. A more refined multifractal spectrum is therefore given by $\mathcal{F}(\alpha, \gamma)=\operatorname{dim}_{H} X_{\alpha, \gamma}$. Our main result is the following.

Theorem. Assume that $g$ and $\alpha \log \left|T^{\prime}\right| \circ \pi$ do not differ by a coboundary and a constant. For sufficiently large $\gamma$ we have that $\mathcal{F}(\alpha, \gamma)=\mathcal{F}(\alpha)$.

Our motivation for this result was an interesting paper by Olsen. In particular, Olsen [10] established a similar result in the special case of self-similar maps and Bernoulli measures. We present an alternative dynamical approach which has the advantage that it extends to $C^{2}$ dynamically defined sets $X$ and Gibbs measures. In section 6 we consider other applications of these results. An integral part of our analysis is the study of the variance of Gibbs measures which is of independent interest.

\section{ITERATED FUNCTION SCHEMES}

We recall the definition and basic facts about iterated function schemes. An iterated function scheme consists of a family $T_{1}, \cdots, T_{k}:[0,1] \rightarrow[0,1]$ of $C^{2}$ contractions. The limit set $X=X\left(T_{1}, \cdots, T_{k}\right)$ is the smallest closed set for which $\cup_{i=1}^{k} T_{i}(X)=X$. We assume that they satisfy the strong separation condition, i.e., the sets $T_{i}(X)$ are pairwise disjoint and $X$ will be a Cantor set. We use the following definitions.

Definition. Let $T: X \rightarrow X$ be the locally expanding map defined by

$$
T(x)=T_{i}^{-1} \text { if } x \in T_{i}(X)
$$

Given $0<\alpha \leq 1$, for $g: X \rightarrow \mathbb{R}$ we write

$$
|g|_{\alpha}=\sup _{x \neq y}\left\{\frac{|g(x)-g(y)|}{|x-y|^{\alpha}}\right\}
$$

For any compact set $Y \subset \mathbb{R}$, the space of $\alpha$-Hölder continuous functions $C^{\alpha}(Y)=$ $\left\{g:|g|_{\alpha}<+\infty\right\}$ is a Banach space with norm $\left.|| g\right|_{\alpha}=|g|_{\alpha}+|g|_{\infty}$.

Definition. Let $p_{i}:[0,1] \rightarrow \mathbb{R}, i=1, \cdots, k$, be Hölder continuous functions such that $\sum_{i=1}^{k} p_{i}(x)=1$ and $0 \leq p_{i}(x) \leq 1$. A measure $\nu$ on $X$ is called self-conformal if there exists such functions $p_{i}$ such that

$$
\left(p_{1} T_{1}^{*} \mu+\cdots p_{k} T_{k}^{*} \nu\right)=\nu
$$


i.e., $\int\left(p_{1}(x) w\left(T_{1} x\right)+\cdots+p_{k}(x) w\left(T_{k} x\right)\right) d \nu(x)=\int w(x) d \nu(x)$, for any continuous function $w: X \rightarrow \mathbb{R}$.

The self-conformal measures fall into a broad class of measures. Let $g: X \rightarrow \mathbb{R}$ be a Hölder continuous function. We define the pressure $P(g)$ of $g$ by

$$
P(g)=\sup \left\{h(T, \mu)+\int g d \mu: \mu=T \text {-invariant probability measure }\right\}
$$

where $h(T, \mu)$ is the entropy of the measure $\mu$ with respect to the transformation $T$.

Definition. A Gibbs measure for $g \in C^{\alpha}(X, \mathbb{R})$ is an invariant probability measure on $T$ such that

$$
\frac{1}{C} \leq \frac{\mu\left(T_{x_{0}} \circ \cdots \circ T_{x_{n}}(X)\right)}{e^{g^{n}(x)-n P(g)}} \leq C
$$

where $g^{n}(x)=g(x)+g(T x)+\cdots+g\left(T^{n-1} x\right)$. The sets $T_{x_{0}} \circ \cdots \circ T_{x_{n}}(X)$ are called cylinders.

A Gibbs measure is always ergodic. If $\mu$ is a Gibbs measure for $g$ then $P(g)=$ $h(T, \mu)+\int g \mathrm{~d} \mu$. A self-conformal measure is necessarily a Gibbs measure with respect to the function $g(x)=\log p_{x_{0}}(T x)$, where $x \in T_{x_{0}}(X)$.

Lemma 1.1. If we assume that $\sum_{i=1}^{k} e^{g\left(T_{i} x\right)}=1$ then we have that

$$
\min _{x}\left\{e^{g^{n}(x)}\right\} \leq \frac{\mu\left(T_{i_{0}} \circ \cdots \circ T_{i_{n-1}}(X)\right)}{e^{-n P(g)}} \leq \max _{x}\left\{e^{g^{n}(x)}\right\}
$$

Given a probability measure $\nu$ we define its Hausdorff dimension to be the infimum of the Hausdorff dimensions of Borel sets of full measure.

Definition. We can define the (symbolic) pointwise dimension at $x \in X$ by

$$
d_{\nu}(x)=\lim _{n \rightarrow+\infty} \frac{\log \nu\left(B\left(x,\left|\left(T^{n}\right)^{\prime}(x)\right|^{-1}\right)\right)}{\log \left|\left(T^{n}\right)^{\prime}(x)\right|^{-1}},
$$

providing the limit exists.

In the case of Gibbs measures $\mu$ the Federer property holds, i.e., there exists $\lambda>1$ and $C_{1}>1$ such that for every $x \in X$ and $r>0$ we have that

$$
\nu(B(x, \lambda r)) \leq C_{1} \nu(B(x, r))
$$

There exists $C_{2}>0$ such that $x, y \in T_{i_{0}} \circ \cdots \circ T_{i_{n-1}} X$ we have that

$$
C_{2}^{-1} \leq \frac{\left|\left(T^{n}\right)^{\prime}(x)\right|}{\left|\left(T^{n}\right)^{\prime}(y)\right|} \leq C_{2} .
$$

Thus for any $x \in T_{i_{1}} \circ \cdots \circ T_{i_{n}} X$ we have that

$$
C_{1}^{-1} \lambda^{-N} \leq \frac{\nu\left(T_{i_{0}} \circ \cdots \circ T_{i_{n-1}} X\right)}{\nu\left(B\left(x,\left|\left(T^{n}\right)^{\prime}(x)\right|^{-1}\right)\right)} \leq C_{1} \lambda^{N}
$$


where $N$ is chosen so that $\lambda^{N}>C_{2}$, and

$$
C_{2}^{-1} \leq \frac{\operatorname{diam}\left(T_{i_{0}} \circ \cdots \circ T_{i_{n-1}} X\right)}{\left|\left(T^{n}\right)^{\prime}(x)\right|^{-1}} \leq C_{2} .
$$

Thus for $\nu$ the (symbolic) pointwise dimension coincides with

$$
d_{\nu}(x)=\lim _{n \rightarrow+\infty} \frac{\log \nu\left(T_{i_{0}} \circ \cdots \circ T_{i_{n-1}} X\right)}{\log \operatorname{diam}\left(T_{i_{0}} \circ \cdots \circ T_{i_{n-1}} X\right)},
$$

and with the usual pointwise dimension. Let $\Sigma=\prod_{0}^{\infty}\{1, \cdots, k\}$ be a full shift space on $k$-symbols and let $\sigma: \Sigma \rightarrow \Sigma$ denote the shift given by $(\sigma x)_{n}=x_{n+1}$. Let $\pi: \Sigma \rightarrow X$ be the natural coding defined by

$$
\pi(x)=\lim _{n \rightarrow+\infty} T_{x_{0}} \cdots T_{x_{n}}(0) .
$$

Example. The simplest case is where there are linear contractions with rates $0<$ $r_{1}, \cdots, r_{k}<1$ and a fixed probability vector $\underline{p}=\left(p_{1}, \cdots, p_{k}\right)$. In this case, $\nu=\pi^{*} \mu_{\underline{p}}$ corresponds to the Bernoulli measure $\mu_{\underline{p}}=\left(p_{1}, \cdots, p_{k}\right)^{\mathbb{Z}^{+}}$on $\Sigma$.

We can define the (symbolic) multifractal spectrum of the measure $\nu$ by

$$
\mathcal{F}(\alpha)=\operatorname{dim}_{H}\left(X_{\alpha}\right)
$$

where $X_{\alpha}=\left\{x: d_{\nu}(x)=\alpha\right\}$. This function has been extensively studied by various authors, notably Pesin and Weiss [14], Ledrappier [9], Cawley and Mauldin [3], Olsen [11]. In [14] the approach taken was to use Gibbs measures and thermodynamic formalism.

Let $\Phi: X \rightarrow \mathbb{R}$ be a Hölder continuous function and $\nu$ the associated Gibbs measure. The multifractal spectrum of $\mu$ is described in [14]. We outline their approach which will be crucial in the rest of this paper. For $q \in \mathbb{R}$ we define

$$
\phi_{q}=-t(q) \log \left|T^{\prime}(x)\right|+q(\Phi+P(\Phi))
$$

where $t(q)$ is chosen to be the unique value such that $P\left(\phi_{q}\right)=0$. Let $\nu_{q}$ be the Gibbs measure associated with $\phi_{q}$. It can be shown that for $\nu_{q}$ almost all $x$, $d_{\nu}(x)=-t^{\prime}(q)$. Since $t^{\prime}(q)$ is a strictly monotone function, we can associate each value of $q$ to a value of $\alpha$ by $\alpha(q)=-t^{\prime}(q)$. Using this method [14] gives the following result.

Proposition 1.1. Let $T_{1}, \ldots, T_{k}:[0,1] \rightarrow[0,1]$ be a conformal $C^{2}$ iterated function scheme satisfying the strong separation condition. Let $X$ be the limit set and a conformal measure $\nu$.

(1) $\mathcal{F}(\alpha)$ is analytic and convex in a neighbourhood of $\alpha_{0}=\operatorname{dim}_{H}(X)$ which we will denote $\left(\alpha_{\min }, \alpha_{\max }\right)$.

(2) For $\alpha \in\left(\alpha_{\min }, \alpha_{\max }\right)$ there exists a measure $\nu_{\alpha}$ such that $\nu_{\alpha}\left(X_{\alpha}\right)=1$ and $d_{\nu}(x)=\alpha$, a.e. $\left(\nu_{\alpha}\right)$. Moreover, $\operatorname{dim} \nu_{\alpha}=\mathcal{F}(\alpha)$.

(3) For $\alpha \in\left(\alpha_{\min }, \alpha_{\max }\right), \mathcal{F}(\alpha)$ is given by the Legendre transform of $t(q)$. That is

$$
\mathcal{F}(\alpha)=\inf _{q}\{\alpha q+t(q)\}
$$

To analyze the finer sets $\mathcal{F}(\alpha, \gamma)$ we will use the statistical properties of Gibbs measures. 


\section{Statistical Properties of Gibbs measures}

We say that $f \in C^{0}(X, \mathbb{R})$ is cohomologous to a constant if there exists $u \in$ $C^{0}(X, \mathbb{R})$ and $c \in \mathbb{R}$ such that $f=u \circ T-u+c$. If $\mu$ is a Gibbs measure then the following analogues of well known statistical theorems hold.

Proposition 2.1 (Law of the Iterated Logarithm) [4]. Let $f$ be a Hölder continuous function with $\int f d \mu=0$. Assume that $f$ is not cohomologous to a constant, then there exists $\gamma>0$ such that for a.e. ( $\mu$ ) $x$ we have that

$$
\frac{f^{n}(x)}{\sqrt{n \log \log n}} \rightarrow \sqrt{2 \gamma} \text {, as } n \rightarrow+\infty \text {. }
$$

Proposition 2.2 (Central Limit Theorem)[1], [4]. Let $f$ be a Hölder continuous function with $\int f d \mu=0$. Assume that $f$ is not cohomologous to a constant, then there exits $\gamma>0$ such that

$$
\lim _{n \rightarrow+\infty} \mu\left\{x \in X: \frac{1}{\sqrt{n}} f^{n}(x) \leq t\right\}=\frac{1}{\sqrt{2 \pi \gamma}} \int_{-\infty}^{t} e^{-\frac{\gamma y^{2}}{2}} d y .
$$

We say that $f$ is a lattice function if there exists $u \in C^{0}(X, \mathbb{R}), c \in \mathbb{R}$ and $\psi \in C^{0}(X, a \mathbb{Z})$, for some $a>0$, such that $f=u \circ X-u+\psi+c$. Generically the functions $f^{n}$ will be non-lattice.

Proposition 2.3 (Local Limit Theorem). Let $f$ be a Hölder continuous function with $\int f d \mu=0$.

(1) Assume that $f$ is a non-lattice function then there exists $\gamma>0$

$$
\lim _{n \rightarrow \infty} \frac{\mu\left\{x \in X: a \leq f^{n}(x) \leq b\right\}}{\frac{1}{\sqrt{n}} \frac{(b-a)}{\sqrt{2 \gamma}}}=1,
$$

[8], [2, Th 9.2].

(2) Assume that $f$ is a lattice function then provided $b-a$ is sufficiently large there exists $C>0$ (depending on $\gamma$ ) such that

$$
\frac{1}{C} \leq \frac{\mu\left\{x \in X: a<f^{n}(x)<b\right\}}{\frac{1}{\sqrt{n}}} \leq C
$$

for all $n$ sufficiently large [2, Thm 9.6].

In these three propositions the value of $\gamma$ is the same.

All of these results are special cases of more general invariance principles [4]. The next lemma relates dynamical properties of the measure $\nu$ to the pointwise (symbolic) dimension of $\nu$ on $X$.

Lemma 2.1. If $\mu$ is a Gibbs measure for a Hölder continuous function $\Phi: X \rightarrow \mathbb{R}$ and $\nu$ is an ergodic measure then

$$
d_{\mu}(x)=\frac{\int \Phi(x) d \nu-P(\Phi)}{\int \log \left|T^{\prime}(x)\right| d \nu} \text { for a.e }(\nu) x
$$


Proof. We first observe that $\log \left|\left(T^{n}\right)^{\prime}\right|=\sum_{i=0}^{n-1} \log \left|T^{\prime}\right|$ and then we can write

$$
\lim _{n \rightarrow+\infty} \frac{1}{n} \log \left|\left(T^{n}\right)^{\prime}(x)\right|=\int \log \left|T^{\prime}\right| d \nu, \text { a.e. }(\nu) x .
$$

The Gibbs property of $\mu$ and the Birkhoff Ergodic Theorem gives that

$$
\begin{aligned}
\lim _{n \rightarrow+\infty} \frac{1}{n} \log \mu\left[x_{0}, \ldots, x_{n-1}\right] & =\lim _{n \rightarrow+\infty} \frac{1}{n}\left(\Phi^{n}(x)-n P(\Phi)\right) \\
& =\int \Phi \mathrm{d} \nu-P(\Phi) \text { for a.e. }(\nu) x .
\end{aligned}
$$

The proof follows from combining (2.1) and (2.2).

Definition. The variance of a Gibbs measure $\nu$ (with respect to the function $g$ : $X \rightarrow \mathbb{R}$ ) and a Hölder function $F: X \rightarrow \mathbb{R}$ is defined to be

$$
\gamma(X, \nu):=\lim _{n \rightarrow+\infty} \frac{1}{n} \int\left(\sum_{i=0}^{n-1} F\left(T^{i} x\right)-\int F d \nu\right)^{2} d \nu(x)
$$

The proof of convergence of this limit, and alternative definitions, appear in [12] and [2]. The variance plays a key role in the following result which start are analysis of the finer multifractal spectrum $\mathcal{F}(\alpha, \gamma)$. It is a simple extension to the non-linear case of Proposition 1.1 in [10].

Lemma 2.4. Let $\mu$ be a self-conformal measure corresponding to a Gibbs measure with potential $\Phi$ which is not cohomologous to a constant. Fix $q \in \mathbb{R}$ and $\alpha=-t^{\prime}(q)$. If $\gamma_{\alpha}$ is the variance of $f(x)=\Phi(x)-P(\Phi)-\alpha \log \left|T^{\prime}(x)\right|$ then $\mathcal{F}(\alpha)=\mathcal{F}\left(\alpha, \sqrt{2 \gamma_{\alpha}}\right)$.

Proof. It can be deduced from the work in [15] that $\int f d \mu=0$. Thus by the Law of the Iterated Logarithm (Proposition 2.1) for $\nu_{\alpha}$ we can write

$$
\limsup _{n \rightarrow \infty} \frac{f^{n}(x)}{\sqrt{n \log \log n}}=\sqrt{2 \gamma_{\alpha}} \text {, for a.e. }\left(\nu_{\alpha}\right) .
$$

This completes the proof.

We would like to replace $\gamma_{\alpha}$ by other values $\gamma$. In order to see this we would like to consider other Gibbs measures $\mu^{\prime}$ (associated to suitable Hölder continuous functions $g^{\prime}: X \rightarrow \mathbb{R}$ ) which satisfy the following properties:

(1) the variance is $\gamma=\gamma\left(\mu^{\prime}\right)$; and

(2) the measure $\mu^{\prime}$ has the same limit

$$
\alpha=\lim _{n \rightarrow+\infty} \frac{\log \nu^{\prime}\left(B\left(x,\left|\left(T^{n}\right)^{\prime}(x)\right|^{-1}\right)\right)}{\log \left|\left(T^{n}\right)^{\prime}(x)\right|^{-1}}=\frac{\left(\int f d \mu^{\prime}-P(f)\right)}{\int \log \left|T^{\prime}\right| d \mu^{\prime}}
$$

for a.e. $\left(\nu^{\prime}\right) x \in X$. In particular we look for Gibbs measures $\mu^{\prime}$ such that $\int f \mathrm{~d} \mu^{\prime}=0$ for the function $f$ defined in Lemma 2.4. This property implies $(2.3)$.

In the next two sections we shall consider this problem in detail. 


\section{Estimating the variance of GibBs measures}

The variance appears in a number of different statistical properties, as we saw in the last section, and so estimating its value is a problem of independent interest. For example, the variance appears in a number of statistical properties of hyperbolic systems (e.g., Propositions 2.1, 2.2 and 2.3). In practice, we shall use a well known characterization of the variance in terms of the second derivative of the pressure [17],[12]. The differentiability of the pressure is most conveniently studied using a characterization in terms of transfer operators. In order to work at a fairly general level, assume that $u, v: X \rightarrow \mathbb{R}$ are any two Hölder continuous functions. Let $C^{\alpha}(X)$ denote the $\alpha$-Holder continuous functions. We can define a transfer operator $\mathcal{L}_{u}: C^{\alpha}(X) \rightarrow C^{\alpha}(X)$ by

$$
\mathcal{L}_{u} h(x)=\sum_{T y=x} e^{u(y)} h(y)
$$

We want to consider two simple normalization hypotheses.

Hypothesis I. Assume that $\mathcal{L}_{u} 1=1$.

Hypothesis II. Assume that $\mathcal{L}_{u} v=0$.

In order to show that these assumptions can be made without any significant loss of generality, first recall that the Gibbs measure and variance are unchanged by adding coboundaries and constants to functions.

Lemma 3.1. Given any $u$ we can find $w \in C^{\alpha}(X)$ such that $u^{\prime}=u+w \circ T-w-$ $P(u)$ satisfies hypothesis $I$.

Proof. This is a standard result [12].

Let $\mu$ denote the unique Gibbs state associated to $u$. If $\mathcal{L}_{u} 1=1$ then $\mathcal{L}_{u}^{*} \mu=\mu$ [12]. We require the following result on the spectrum of $\mathcal{L}_{u}: C^{\alpha}(X) \rightarrow C^{\alpha}(X)$.

Lemma 3.2. The eigenvalue 1 for $\mathcal{L}_{u}$ is simple. Moreover, the spectral radius of the operator $\mathcal{L}-\mu: C^{\alpha}(X) \rightarrow C^{\alpha}(X)$ is strictly smaller than 1 . More precisely, there exist $0<\rho<1$ and $C>0$ such that $\left\|\mathcal{L}_{u}^{n} v\right\|_{\alpha} \leq C \rho^{n}\|v\|_{\alpha}$, for all $v \in$ $C^{\alpha}(X)$ and $n \geq 1$.

Proof. The proof appears, for example, in [12]

We can now this lemma to prove the following.

Lemma 3.3. Assume hypothesis I. Then given any $v$ with $\int v d \mu=0$ we can find $r \in C^{\alpha}(X)$ such that $v^{\prime}=v-r T+r$ satisfies hypothesis II.

Proof. We can define $r=\sum_{n=1}^{\infty} \mathcal{L}_{u}^{n} v$. This converges to a function in $C^{\alpha}(X)$, because of the spectral properties of the operator $\mathcal{L}-\mu$ described in Lemma 3.2. Since $\mathcal{L}_{u} 1=1$ we have that $\mathcal{L}_{u} U_{T}=I$, where $U_{T} v=v \circ T$. In particular, $\mathcal{L}_{u} v^{\prime}=$ $\mathcal{L}_{u} v+\mathcal{L}_{u}\left(r-U_{T} r\right)$, but since $\mathcal{L}_{u}\left(r-U_{T} r\right)=\mathcal{L}_{u} r-r=-\mathcal{L}_{u} v$, by construction, we see that $\mathcal{L}_{u} v=0$. 
Lemma 3.4. Under hypothesis I and hypothesis II we have that $\gamma=\int v^{2} d \mu$.

Proof. Let $\lambda(u)$ be the maximal eigenvalue of the operator $\mathcal{L}_{u}$. The variance $\gamma$ is also characterized by $\gamma=\frac{d^{2} \lambda(u+t v)}{d t^{2}}[12]$. This essentially follows from perturbation theory on the eigenvalue equation $\mathcal{L}_{u+t v} h(t)=\lambda(t) h(t)$. The first derivative of both sides of this identity gives

$$
\mathcal{L}_{u+t v}\left(v h(t)+h^{\prime}(t)\right)=\lambda^{\prime}(t) h(t)+\lambda(t) h^{\prime}(t) .
$$

When $t=0$, we have $\lambda^{\prime}(0)=0$ and $\lambda(0)=1$ and so

$$
\mathcal{L}_{u}\left(h^{\prime}(0)\right)=\mathcal{L}_{u}\left(v+h^{\prime}(0)\right)=h^{\prime}(0)
$$

Since 1 is a simple eigenvalue for $\mathcal{L}_{u}$, with constant eigenfunction, we deduce that $h^{\prime}(0)$ is a constant function. The second derivative of both sides of the identity gives

$$
\mathcal{L}_{u+t v}\left(v^{2} h(t)+2 v h^{\prime}(t)+h^{\prime \prime}(t)\right)=\lambda^{\prime \prime}(t) h(t)+2 \lambda^{\prime}(t) h^{\prime}(t)+\lambda(t) h^{\prime \prime}(t) .
$$

We can evaluate this second expression at $t=0$. We can then integrate both sides with respect to $\mu$, and since $\mu=\mathcal{L}_{u}^{*} \mu$ we have that

$$
\begin{aligned}
& \mu\left(v^{2} h(0)\right)+2 \mu\left(v h^{\prime}(0)\right)+\mu\left(h^{\prime \prime}(0)\right) \\
& =\lambda^{\prime \prime}(0) \mu(h(0))+2 \lambda^{\prime}(0) \mu\left(h^{\prime}(0)\right)+\lambda(0) \mu\left(h^{\prime \prime}(0)\right) .
\end{aligned}
$$

Since $\lambda(0)=1$ we can cancel the last terms on each side. By hypothesis II, we know (by considering the expression (3.1)) that $h^{\prime}(0)=0$, which eliminates an extra term on each side of (3.2) and leaves the identity

$$
\lambda^{\prime \prime}(0)=\frac{\mu\left(v^{2} h(0)\right)}{\mu(h(0))} .
$$

However, by hypothesis I we have that $h(0)=1$ and, by the usual normalization, $\mu(h(0))=1$. This gives the result.

Unfortunately, we first have to accept the following limitation if we consider only $T$-invariant probabilities.

Corollary. Under hypotheses I and II we can bound

$$
(\inf v)^{2} \leq \gamma=\lambda^{\prime \prime}(0) \leq(\sup v)^{2}
$$

If we don't assume hypotheses I and II we get a similar result. Applying Lemmas 3.1 and 3.3, changes the function $v$ by at most a coboundary and a constant. Furthermore, the variance is unchanged by adding a coboundary and a constant (since the pressure is unchanged by adding coboundaries to $u$ and $v$ ).

Example. We can consider the special case of locally constant functions $u$ and $v$ which are constant on each inverse branch $T_{i}(X)$ and a self-similar iterated function 
system. In this case the Gibbs measure $\mu$ for $u$ is a projection of Bernoulli measure on the Bernoulli shift $\Sigma$ associated to a vector $\underline{p}=\left(p_{1}, \cdots, p_{k}\right)$, where

$$
p_{i}=\frac{e^{-u_{i}}}{e^{-u_{1}}+\cdots+e^{-u_{k}}}
$$

and $u_{i}$ is the value $u(x)$ which takes on $T_{i}(X)$. Assuming hypothesis II, the variance in this case can be calculated to be

$$
\gamma=\sum_{i=1}^{k} p_{i} v_{i}^{2}
$$

where $v_{i}$ is the value $v(x)$ which takes on $T_{i}(X)$.

Returning to the application we are interested in, the corollary suggests that we need to look at a broader class of measures. More precisely, for $n \geq 2$ we shall consider the probability measures on $X$ which are $T^{n}$-invariant, rather than the more restrictive assumption of being $T$-invariant.

\section{INVARIANT MEASURES FOR $T^{n}$}

Given $\alpha, \gamma>0$, we want to consider invariant measures $\nu_{\alpha, \gamma}$ for which the local dimension $d_{\nu}(x)$ at almost all points is the same with respect to either $\nu_{\alpha}$ or $\nu_{\alpha, \gamma}$. The measure $\nu_{\alpha, \gamma}$ can be used to give a lower bound on $\operatorname{dim}_{H}\left(X_{\alpha, \gamma}\right)$. However, we see from the previous section that it is not enough to consider $T$-invariant Gibbs measures and we need to consider $T^{n}$-invariant Gibbs measures, for $n \geq 2$.

Definition. Let $\mathcal{M}^{n}$ denote the space of $T^{n}$-invariant Gibbs measures on $X$.

Clearly, the $T$-invariant measures $\mu$ are also contained in $\mathcal{M}^{n}$. The next lemma compares the variances for these two points of view.

Lemma 4.1. Let $f$ be a Hölder continuous function and let $\mu$ be a T-invariant Gibbs measure.

(1) $h\left(T^{n}, \mu\right)=n h(T, \mu)$

(2) If $\gamma\left(T^{n}, \mu, f^{n}\right)$ is the variance for the function $f^{n}$ with respect to $T^{n}$ and $\mu$ then $\gamma(T, \mu, f)=\frac{1}{n} \gamma\left(T^{n}, \mu, f^{n}\right)$

Proof. The first part is Abramov's Theorem. For the second part, we observe that

$$
\begin{aligned}
\gamma(T, \mu, f)=\operatorname{var}(\phi, \mu): & =\lim _{k \rightarrow+\infty} \frac{1}{k} \int\left(\sum_{i=0}^{k-1} f\left(T^{i} x\right)-\int f d \mu\right)^{2} d \mu(x) \\
& =\lim _{k \rightarrow+\infty} \frac{1}{n k} \int\left(\sum_{i=0}^{n k-1} f\left(T^{i} x\right)-\int f d \mu\right)^{2} d \mu(x) \\
& =\frac{1}{n} \lim _{k \rightarrow+\infty} \frac{1}{k} \int\left(\sum_{i=0}^{n k-1} f^{k}\left(T^{n i} x\right)-\int f d \mu\right)^{2} d \mu(x) \\
& =\frac{1}{n} \gamma\left(T^{n}, \mu, f^{n}\right)
\end{aligned}
$$

This completes the proof.

Considering $\mu$ as an element of $\mathcal{M}^{n}$ leads to a similar formulation of the variance. 
Lemma 4.2. Let $\mu \in \mathcal{M}$ be a Gibbs measure with potential g satisfying hypothesis $I$ (with respect to $T$ ). Assume that $f$ satisfies hypothesis II then it corresponds to a measure in $\mathcal{M}$ with potential $g^{n}$ satisfying hypothesis $I$ (with respect to $T^{n}$ ) and $f^{n}$ satisfies hypothesis II.

Proof. Let us denote by $\mathcal{L}_{g, T}$ and $\mathcal{L}_{g^{n}, T^{n}}$ the transfer operators in each case. Since $\mathcal{L}_{g, T}(h \circ T)=h$ and $\mathcal{L}_{g^{n}, T^{n}}=\mathcal{L}_{g, T}^{n}$ we see that $\mathcal{L}_{g^{n}, T^{n}} 1=1$ and $\mathcal{L}_{g^{n}, T^{n}} f^{n}=0$, i.e., they satisfy hypothesis I and II (with respect to $T^{n}$ ).

We can use the following estimates to arrange the variance to be higher. If $\underline{i}$ is a finite word and $x \in X$ then we let $T_{\underline{i}} x=T_{i_{0}} \circ \cdots \circ T_{i_{n-1}}(x)$.

Lemma 4.3. There exist $D, E>0$, such that for all $n \geq 1$ and $x, y$ we can bound for $|\underline{i}|=n$

$$
\left|f^{n}\left(T_{\underline{i}} x\right)-f^{n}\left(T_{\underline{i}} y\right)\right| \leq D \text { and }\left|g^{n}\left(T_{\underline{i}} x\right)-g^{n}\left(T_{\underline{i}} y\right)\right| \leq E .
$$

Proof. We can bound

$$
\begin{aligned}
\left|f^{n}\left(T_{\underline{i}} x\right)-f^{n}(\underline{i} y)\right| & \leq \sum_{k=0}^{n-1}\left|f\left(T^{k} T_{\underline{i}} x\right)-f\left(T^{k} T_{\underline{i}} y\right)\right| \\
& \leq \sum_{k=0}^{n-1} C \theta^{\alpha(n-k)} \\
& \leq \frac{C \theta}{1-\theta}=D
\end{aligned}
$$

where $\theta=\max _{i}\left\{\left\|T_{i}^{\prime}\right\|_{\infty}\right\}<1$ and $\alpha, C>0$ are constants coming from the Hölder continuity of $f$. Similarly, we can bound the expression for $g$.

From the definition of a Gibbs measure $\mu$ for $g$ with $P(g)=0$, we have the following result.

Corollary. For any cylinder of length $n$ we can bound

$$
e^{g^{n}\left(T_{\underline{i}} x\right)-E} \leq \mu\left(T_{\underline{i}} X\right) \leq e^{g^{n}\left(T_{\underline{i}} x\right)+E}
$$

The following lemma will prove useful later.

Lemma 4.4. Assume that $\int f d \mu=0$. If $f$ is not a coboundary, then we can find periodic points $T^{m} x=x$ and $T^{m} x^{\prime}=x^{\prime}$ such that $f^{m}(x)<0$ and $f^{m}\left(x^{\prime}\right)>0$.

Proof. By Livsic's theorem, we know that $f^{m}(x)=0$ whenever $T^{m} x=x$ is equivalent to $f$ being a coboundary [12]. If $f$ is not a coboundary, then there must be either a periodic point $T^{m} x=x$ such that $T^{m}(x)>0$ or a periodic point $T^{m} x^{\prime}=x^{\prime}$ such that $T^{m}\left(x^{\prime}\right)<0$. However, it is easy to see that both cases must exist simultaneously. We then take the least common multiple to complete the proof.

We can assume without loss of generality that $T x=x$ and $T x^{\prime}=x^{\prime}$ are both fixed points. Let us denote by $\delta=f(x)>0$ and $\delta^{\prime}=f\left(x^{\prime}\right)<0$.

Notation. Given $n \geq 1$, let $j$ denote the word of length $n$ corresponding to the cylinder containing $x$. Let $\underline{k}$ denote the word of length $n$ corresponding to the cylinder containing $x^{\prime}$.

Definition. Let $G_{\epsilon}(n)$ be the set of cylinders $\underline{i}$ containing at least one point $y$ for which $\left|f^{n}(y)\right| \leq \epsilon$. 
Lemma 4.5. There exists $C_{1}>0$ such that

$$
\frac{1}{C_{1} \sqrt{n}} \leq \mu\left(G_{\epsilon}(n)\right) \leq \frac{C_{1}}{\sqrt{n}}
$$

for all $n \geq 1$.

Proof. By the Local Limit Theorem (Proposition 2.3) we can deduce that there exists $C \geq 0$

$$
\frac{1}{C \sqrt{n}} \leq \mu\left\{x:\left|f^{n}(x)\right|<\epsilon\right\} \leq \frac{C}{\sqrt{n}}
$$

for all $n$ sufficiently large. We can cover the set by cylinders $C_{i}$ of length $n$ which contain points $x_{i} \in C_{i}$ for which $f^{n}\left(x_{i}\right)<\epsilon$. Recall that by Lemma 4.3 we can bound $\left|f^{n}(x)-f^{n}(y)\right| \leq D$, for $x, y \in C_{i}$ (where $D$ is independent of $n$ ). Thus we see that

$$
\cup_{i} \mu\left(C_{i}\right) \leq \mu\left\{x:\left|f^{n}(x)\right|<\epsilon+D\right\}
$$

This completes the proof.

We can define for each word $\underline{i}$ of length $n$

$$
\alpha_{\underline{i}}(x)=\frac{f^{n}\left(T_{\underline{i}} x\right)-f^{n}\left(T_{\underline{k}} x\right)}{f^{n}\left(T_{\underline{j}} x\right)-f^{n}\left(T_{\underline{k}} x\right)} .
$$

As $n \rightarrow+\infty$, the terms $\alpha_{\underline{\underline{i}}}(x)$ converges to $\frac{\delta^{\prime}}{\delta+\delta^{\prime}}$. For every $\beta \in[0,1]$ we want to replace $e^{g^{n}(x)}$ by a new weight function $e^{\bar{g}_{n}^{(\beta)}(x)}$ defined by

$$
\begin{aligned}
& e^{\bar{g}_{n}^{(\beta)}\left(T_{\underline{i}} x\right)}=(1-\beta) e^{\bar{g}_{n}\left(T_{\underline{i}} x\right)} \text { if } T_{\underline{i}} \in G_{\epsilon}(n) \\
& e^{\bar{g}_{n}^{(\beta)}\left(T_{\underline{j}} x\right)}=e^{g^{n}\left(T_{\underline{j}} x\right)}+\beta \sum_{\underline{i} \in G_{\epsilon}} \alpha_{\underline{i}}(x) e^{g^{n}\left(T_{\underline{\underline{i}}} x\right)} \\
& e^{{\overline{\bar{g}_{n}}}^{(\beta)}\left(T_{\underline{k}} x\right)}=e^{g^{n}\left(T_{\underline{k}} x\right)}+\beta \sum_{T_{\underline{\underline{i}}} \in G_{\epsilon}}\left(1-\alpha_{\underline{i}}(x)\right) e^{g^{n}\left(T_{\underline{\underline{x}}} x\right)}
\end{aligned}
$$

and for all of the other inverse branches there is no change from $e^{g^{n}(x)}$.

Lemma 4.6. The transfer operator $\mathcal{L}_{\bar{g}_{n}^{(\beta)}}$ for $\bar{g}_{n}^{(\beta)}$ and $T^{n}$ satisfies

$$
\mathcal{L}_{\bar{g}_{n}^{(\beta)}} 1=1 \text { and } \mathcal{L}_{\bar{g}_{n}^{(\beta)}} f^{n}=0
$$

Proof. Both of these properties follow from the definition of $\bar{g}_{n}^{(\beta)}$. For the first part simply observe that

$$
L_{\bar{g}_{n}^{(\beta)}} 1-L_{g^{n}} 1=-\beta \sum_{\underline{i} \in G_{\epsilon}} e^{g^{n}(\underline{i} x)}+\alpha_{\underline{i}}(x) e^{g^{n}\left(T_{\underline{i}} x\right)}+\left(1-\alpha_{\underline{i}}(x)\right) e^{g^{n}\left(T_{\underline{i}} x\right)}=0 .
$$

For the second equality we fix $x$ and note that

$$
\alpha_{\underline{i}}(x) f^{n}\left(T_{\underline{j}} x\right)+\left(1-\alpha_{\underline{i}}(x)\right) f^{n}\left(T_{\underline{k}} x\right)=f^{n}\left(T_{\underline{i}} x\right) .
$$


We then have

$$
\begin{aligned}
& L_{\bar{g}_{n}^{(\beta)}} f^{n}(x)-L_{g^{n}} f^{n}(x) \\
& =-\beta \sum_{\underline{i} \in G_{\epsilon}} e^{g^{n}\left(T_{\underline{i}} x\right)} f^{n}\left(T_{\underline{i}} x\right)+\alpha_{\underline{i}}(x) e^{g^{n}\left(T_{\underline{i}} x\right)} f\left(T_{\underline{j}} x\right)+\left(1-\alpha_{\underline{i}}(x)\right) e^{g^{n}\left(T_{\underline{i}} x\right)} f\left(T_{\underline{k}} x\right) \\
& =-\beta \sum_{\underline{i} \in G_{\epsilon}} e^{g^{n}\left(T_{\underline{i}} x\right)} f^{n}\left(T_{\underline{i}} x\right)+\beta \sum_{\underline{i} \in G_{\epsilon}} e^{g^{n}\left(T_{\underline{i}} x\right)} f^{n}\left(T_{\underline{i}} x\right)=0
\end{aligned}
$$

which completes the proof. that

Let $\bar{\mu}_{\beta}$ be the unique measure such that $\mathcal{L}_{\bar{g}_{n}^{(\beta)}}^{*} \bar{\mu}_{\beta}=\bar{\mu}_{\beta}$. By construction we have

$$
\begin{aligned}
\bar{\mu}_{\beta}\left[T_{\underline{j}}(X)\right] & \geq \min _{x}\left\{e^{\overline{g_{n}^{(\beta)}}\left(T_{\underline{j}} x\right)}\right\} \\
& \geq \min _{x}\left\{e^{g^{n}\left(T_{\underline{j}} x\right)}+\beta \sum_{\underline{i} \in G_{\epsilon}} \alpha_{\underline{i}}(x) e^{g^{n}\left(T_{\underline{i}} x\right)}\right\} \\
& \geq \alpha_{0} \beta \sum_{\underline{i} \in G_{\epsilon}} \min \left\{e^{g^{n}\left(T_{\underline{i}} x\right)}\right\} \\
& \geq \alpha_{0} \beta e^{-2 E} \sum_{\underline{i} \in G_{\epsilon}} \mu\left[T_{\underline{i}}(X)\right] \\
& \geq \frac{\alpha_{0} \beta}{C_{1} e^{2 E}} \frac{1}{\sqrt{n}}
\end{aligned}
$$

by Lemma 4.5. We can estimate

$$
\int\left(f^{n}\right)^{2} d \bar{\mu} \geq(\delta n-D)^{2} \bar{\mu}\left[T_{\underline{j}} X\right]+\left(\delta^{\prime} n+D\right)^{2} \bar{\mu}\left[T_{\underline{k}}\right]
$$

This brings us to the following.

Proposition 4.1. Let $f$ be a Hölder continuous function not cohomologous to 0 and $\mu$ be a Gibbs measure with $\int f d \mu=0$ and variance $\gamma_{\alpha}$. Let $\gamma>\gamma_{0}$. We can choose $n \geq 1$ and a measure $\bar{\mu}$ such that

(i)

$$
\int f^{n} d \bar{\mu}=0
$$

$$
\frac{1}{n} \int\left(f^{n}\right)^{2} d \bar{\mu}=\gamma
$$

$$
\left|\operatorname{dim}_{H}(\mu)-\operatorname{dim}_{H}(\bar{\mu})\right|<\epsilon
$$

Proof. The first part follows from Lemma 4.6. The third part is based on the identities $\operatorname{dim}_{H}(\mu)=h(\mu) / \int \log \left|T^{\prime}\right| d \mu$ and $\operatorname{dim}_{H}(\bar{\mu})=h(\bar{\mu}) / \int \log \left|T^{\prime}\right| d \bar{\mu}$ (cf. [6]), which one sees are arbitrarily close for $n$ sufficiently large. We prove part (ii) in two steps. Firstly we fix $\gamma>\gamma_{0}$ it can be seen from equation (4.1) that it is possible to find $n \geq 1, \beta \in[0,1]$ and $\bar{\mu}_{\beta}$ a $T^{n}$ invariant measure such that

$$
\frac{1}{n} \int\left(f^{n}\right)^{2} \mathrm{~d} \bar{\mu}_{\beta} \geq \gamma
$$


The second part is to show that the map $h:[0,1] \rightarrow \mathbb{R}$ given by

$$
h(\beta)=\frac{1}{n} \int\left(f^{n}\right)^{2} \mathrm{~d} \bar{\mu}_{\beta}
$$

is continuous. Since $\frac{1}{n} \int\left(f^{n}\right)^{2} \mathrm{~d} \bar{\mu}_{0}=\gamma_{0}$ the result then follows by the intermediate value theorem. Since $\mathcal{L}_{\bar{g}_{n}^{(\beta)}} \bar{\mu}_{\beta}=\bar{\mu}_{\beta}$ and $\mu_{\beta}$ is a simple eigenvector for an isolated eigenvalue by perturbation theory $\beta \rightarrow \bar{\mu}_{\beta}$ is continuous in the weak* topology $([17],[12])$. The continuity of $h$ is then immediate.

\section{Proof of TheOrem 1}

We are now in a position to prove Theorem 1 . Let $\mu$ be a self-conformal measure corresponding to a Gibbs measure with potential $\phi$ and an expanding map $T$. We fix $\alpha$ in the region where $\mathcal{F}(\alpha)$ is defined. Let

$$
f(x)=\phi(x)-P(\phi)-\alpha \log \left|T^{\prime}(x)\right|
$$

The conditions imposed on $\phi$ in the statement of the theorem imply that $f$ is not cohomologous to a constant. It follows from [14], [13] that we can find a Gibbs measure $\nu_{\alpha}$ with potential $g$ such that $\operatorname{dim} \nu_{\alpha}=\mathcal{F}(\alpha)$ and $\int f \mathrm{~d} \nu_{\alpha}=0$ hence giving $d_{\mu}(x)=\alpha$ for $\nu_{\alpha}$ almost every $x$ by Birkhoff's Ergodic Theorem. The following Lemma also holds.

Lemma 5.1. By adding a coboundary, if necessary, we can assume that $L_{g} f=0$ and there exist fixed points $x$ and $x^{\prime}$ such that $f(x)>0$ and $f\left(x^{\prime}\right)<0$.

Proof. The first part follows from Lemma 3.1 and Lemma 3.2. The second part follows from Lemma 4.4

Let $\gamma_{0}$ be the variance of $f$ with respect to $\nu_{\alpha}$. Fix $\gamma>\gamma_{0}$. All the conditions for Proposition 4.1 are satisfied so for any $\epsilon>0$ we can choose $n \geq 1$ and a $T^{n}$ invariant ergodic probability measure $\overline{\nu_{\alpha}}$ such that

$$
\int f^{n} d \bar{\nu}_{\alpha}=0
$$

$$
\frac{1}{n} \int\left(f^{n}\right)^{2} d \overline{\nu_{\alpha}}=\gamma
$$

$$
\left|\operatorname{dim}_{H}\left(\nu_{\alpha}\right)-\operatorname{dim}_{H}\left(\overline{\nu_{\alpha}}\right)\right|<\epsilon .
$$

It follows by the law of the iterated logarithm that

$$
\limsup _{k \rightarrow \infty} \frac{f^{n k}(x)}{\sqrt{k \log \log k}}=\sqrt{2 n \gamma}
$$

for $\overline{\nu_{\alpha}}$ almost all $x$. Let $l=n k+m$ where $0 \leq m \leq n-1$ and we have that

$$
\frac{f^{l}(x)}{\sqrt{l \log \log l}}=\frac{f^{n k+m}(x)}{\sqrt{(n k+m) \log \log (n k+m)}}=\frac{f^{n k}(x)+f^{m}\left(T^{n k}(x)\right)}{\sqrt{(n k+m) \log \log (n k+m)}} .
$$


Since $f^{n}(x)$ is uniformly bounded we then have that

$$
\limsup _{l \rightarrow \infty} \frac{f^{l}(x)}{\sqrt{l \log \log l}}=\limsup _{k \rightarrow \infty} \frac{f^{n k}(x)}{\sqrt{n k \log \log (n k)}}=\frac{1}{\sqrt{n}} \limsup _{k \rightarrow \infty} \frac{f^{n k}(x)}{\sqrt{k \log \log k}} .
$$

Thus

$$
\limsup _{l \rightarrow \infty} \frac{f^{l}(x)}{\sqrt{l \log \log l}}=\sqrt{2 \gamma}
$$

This suffices to complete the proof of Theorem 1 .

\section{Subshifts And Horseshoes}

We can use the same method to study subshifts of finite type. Let $A$ denote a $k \times k$ matrix with entries 0 and 1 . Assume that $A$ is irreducible. Let us denote the one-sided shift space by

$$
\Sigma^{+}=\left\{\underline{x}=\left(x_{n}\right) \in\{1, \cdots, k\}^{\mathbb{Z}^{+}}: A\left(x_{i}, x_{i+1}\right)=1\right\} .
$$

Let $\sigma: \Sigma^{+} \rightarrow \Sigma^{+}$be the one sided subshift of finite type defined by $(\sigma x)_{n}=x_{n+1}$.

A function $w: \Sigma^{+} \rightarrow \mathbb{R}$ is called Hölder continuous if there exists $0<\theta<1$ such that

$$
\|w\|_{\theta}:=\sup \left\{\frac{|w(x)-w(y)|}{\theta^{n}}: x_{i}=y_{i} \text {, for } 0 \leq i \leq n-1\right\} \text {. }
$$

Let $\left[x_{0}, \cdots, x_{n-1}\right]=\left\{\underline{y} \in \Sigma^{+}: x_{i}=y_{i}, 0 \leq i \leq n-1\right\}$. The sets $\left[x_{0}, \cdots, x_{n}\right]$ are called cylinders. A Gib̄bs measure for a Hölder continuous function $g: \Sigma^{+} \rightarrow \mathbb{R}$ is an invariant probability measure on $\Sigma^{+}$if there exists $C>0$ and $P=P(f)$ such that

$$
\frac{1}{C} \leq \frac{\mu\left(\left[x_{0}, \cdots, x_{n-1}\right]\right)}{e^{g^{n}(x)-n P(g)}} \leq C,
$$

for all $n \geq 1$, where $g^{n}(x)=g(x)+g(\sigma x)+\cdots+g\left(\sigma^{n-1} x\right)$. By analogy with our previous definitions, we can define the variance of $\mu$ and a Hölder function $F: \Sigma^{+} \rightarrow \mathbb{R}$ by

$$
\operatorname{var}(\phi, \mu):=\lim _{n \rightarrow+\infty} \frac{1}{n} \int\left(\sum_{i=0}^{n-1} F\left(\sigma^{i} x\right)-\int F d \mu\right)^{2} d \mu(x)
$$

The proof of the main theorem for iterated function schemes gives the following variant for one sided subshifts of the law of the iterated logarithm.

Proposition 6.1 (Law of the Iterated Logarithm for one sided subshifts). Let $\mu$ be a $\sigma$ invariant Gibbs measure such that $\int \phi d \mu=0$. Let $\gamma_{0}=\sqrt{2 \operatorname{var}(\phi, \mu)}$. For every $\gamma>\gamma_{0}$ we have that

$$
\operatorname{dim}_{H}\left\{x: \limsup _{n} \frac{\phi^{n}(x)}{\sqrt{n \log \log n}}=\gamma\right\} \geq \operatorname{dim}_{H} \mu
$$

In [15] for a Hölder, non-constant function $\phi: \Sigma \rightarrow \mathbb{R}$ Pesin and Weiss consider the set

$$
B_{\alpha}=\left\{x \in \Sigma: \lim _{n \rightarrow \infty} \frac{\phi^{n}(x)}{n}=\alpha\right\} .
$$


It is shown in [14] that for any $\alpha$ in an open interval, $\left(\alpha_{\min }, \alpha, \max \right)$, we have that there exists a Gibbs measure $\nu_{\alpha}$ such that $\nu_{\alpha}\left(B_{\alpha}\right)=1$ and $\operatorname{dim} B_{\alpha}=\operatorname{dim} \nu_{\alpha}$. It follows that if we let $f=\phi-\alpha$ then $\int f \mathrm{~d} \nu_{\alpha}=0$. Thus we can use Proposition 6.1 to study the properties of the finer sets

$$
B_{\alpha}(\gamma)=\left\{x: \limsup _{n \rightarrow \infty} \frac{\phi^{n}(x)-n \alpha}{\sqrt{n \log \log n}}=\gamma\right\}
$$

We then have the following corollary to Proposition 6.1.

Corollary. Let $\gamma_{\alpha}=\operatorname{var}\left(f, \nu_{\alpha}\right)$. For $\gamma \geq \gamma_{\alpha}$ we have that

$$
\operatorname{dim} B_{\alpha}(\gamma)=\operatorname{dim} B_{\alpha}
$$

We note that it can be easily deduced from the work of Fan and Schmeling, [7] that $\operatorname{dim} B_{\alpha}(0)=\operatorname{dim} B_{\alpha}$ and $\operatorname{dim} B_{\alpha}(\infty)=\operatorname{dim} B_{\alpha}$.

Let us next define the two sided shift space by

$$
\Sigma=\left\{\underline{x}=\left(x_{n}\right) \in\{1, \cdots, k\}^{\mathbb{Z}}: A\left(x_{i}, x_{i+1}\right)=1, \text { for } i \in \mathbb{Z}\right\}
$$

Let $\sigma: \Sigma \rightarrow \Sigma$ be the two sided subshift of finite type defined by $(\sigma x)_{n}=x_{n+1}$. In this case we say that a function $w: \Sigma \rightarrow \mathbb{R}$ is Hölder continuous if there exists $0<\theta<1$ such that

$$
\|w\|_{\theta}:=\sup \left\{\frac{|w(x)-w(y)|}{\theta^{n}}: x_{i}=y_{i}, \text { for }-(n-1) \leq i \leq n-1\right\} \text {. }
$$

We have the corresponding version of the law of the iterated logarithm for two sided shifts.

Proposition 6.2 (Law of the Iterated Logarithm for two sided subshifts). Let $\mu$ be a $\sigma$ invariant Gibbs measure such that $\int \phi d \mu=0$. Let $\gamma_{0}=\sqrt{2 \operatorname{var}(\phi, \mu)}$. For every $\gamma>\gamma_{0}$ we have that

$$
\operatorname{dim}_{H}\left\{x: \limsup _{n} \frac{\phi^{n}(x)}{\sqrt{n \log \log n}}=\gamma\right\} \geq \operatorname{dim}_{H} \mu
$$

We can reduce the proof for two sided shifts to that for one sided subshifts. We recall the following useful results.

Lemma 6.1. Given a Hölder continuous function $\phi: \Sigma \rightarrow \Sigma$ there exists a (Hölder) continuous function $u: \Sigma \rightarrow \mathbb{R}$ such that $\phi^{\prime}(x)=\phi(x)+u(\sigma x)-u(x)$ satisfies $\left(\phi^{\prime}\right)^{n}(x)=\left(\phi^{\prime}\right)^{n}(y)$, if $x, y \in \Sigma$ satisfy $x_{i}=y_{i}$, for $i \geq 0$ [12].

In particular, $\phi^{\prime}$ can be identified with a function on the one sided shift space $\Sigma$. We can replace $\phi$ by $\phi^{\prime}$ without loss of generality.

Lemma 6.2. There exists $C>0$, such that if $x, y$ satisfy $x_{i}=y_{i}$, for $i \geq 0$ then $\left|\phi^{n}(x)-\phi^{n}(y)\right| \leq C$, for each $n \geq 0$.

Proof. Since $\left|\phi\left(\sigma^{i} x\right)-\phi\left(\sigma^{i} y\right)\right| \leq\|\phi\|_{\theta} \theta^{i}$ for $0 \leq i \leq n-1$ we have that $\mid \phi^{n}(x)-$ $\phi^{n}(y) \mid \leq \frac{\|\phi\|_{\theta}}{1-\theta}$. 
Proof of Proposition 6.2. We begin by observing that by Lemma 6.2 if $x, y$ satisfy $x_{i}=y_{i}$, for $i \geq 0$ then

$$
\limsup _{n} \frac{\phi^{n}(x)}{\sqrt{n \log \log n}}=\limsup _{n} \frac{\phi^{n}(y)}{\sqrt{n \log \log n}} .
$$

In particular,

$$
\begin{aligned}
& \operatorname{dim}_{H}\left\{x \in \Sigma: \limsup _{n} \frac{\phi^{n}(x)}{\sqrt{n \log \log n}}=\gamma\right\} \\
& =\operatorname{dim}_{H}\left\{\xi \in \Sigma^{+}: \limsup _{n} \frac{\phi^{n}(\xi)}{\sqrt{n \log \log n}}=\gamma\right\}+\operatorname{dim}_{H} \Sigma^{-} \\
& =\operatorname{dim}_{H}\left\{\xi \in \Sigma^{+}: \limsup _{n} \frac{\phi^{n}(\xi)}{\sqrt{n \log \log n}}=\gamma\right\}+\frac{h(\sigma)}{\log \theta},
\end{aligned}
$$

and

$$
\begin{aligned}
& \operatorname{dim}_{H}\left\{x \in \Sigma: \limsup _{n} \frac{\phi^{n}(x)}{\sqrt{n \log \log n}}=\gamma_{0}\right\} \\
& =\operatorname{dim}_{H}\left\{\xi \in \Sigma: \limsup _{n} \frac{\phi^{n}(\xi)}{\sqrt{n \log \log n}}=\gamma_{0}\right\}+\operatorname{dim}_{H} \Sigma^{-} \\
& =\operatorname{dim}_{H}\left\{\xi \in \Sigma: \limsup _{n} \frac{\phi^{n}(\xi)}{\sqrt{n \log \log n}}=\gamma_{0}\right\}+\frac{h(\sigma)}{\log \theta},
\end{aligned}
$$

where

$$
\Sigma^{-}=\left\{\underline{x}=\left(x_{n}\right) \in \prod_{-\infty}^{-1}\{1, \cdots, k\}: A\left(x_{i}, x_{i+1}\right)=1 \text { for } i<-1\right\}
$$

and $\operatorname{dim}_{H} \Sigma^{-}=\frac{h(\sigma)}{\log \theta}$. The result then follow from Proposition 6.1.

Finally, we consider a more geometric setting. Let $f: \Lambda \rightarrow \Lambda$ be a $C^{2}$ Smale horseshoe and let $\Phi: \Lambda \rightarrow \mathbb{R}$ be a Hölder continuous function which is not cohomologous to a constant. The definitions of Gibbs measures and variance are in complete analogy with those given before. We have the following related result.

Proposition 6.3 (Law of the Iterated Logarithm for horseshoes). Let $\mu$ be a Gibbs measure such that $\int \Phi d \mu=0$. Let $\gamma_{0}=\sqrt{2 \operatorname{var}(\Phi, \mu)}$. For any $\gamma>\gamma_{0}$ we have that

$$
\operatorname{dim}_{H}\left\{x: \limsup _{n} \frac{\Phi^{n}(x)}{\sqrt{n \log \log n}}=\gamma\right\} \geq \operatorname{dim}_{H} \mu
$$

Proof. We can model $f$ by a two sided subshift $\sigma: \Sigma \rightarrow \Sigma$. By choosing a Markov Partition $\mathcal{P}=\left\{T_{1}, \cdots, T_{k}\right\}$ we can associate a continuous semi-conjugacy map $\pi: \Sigma \rightarrow \Lambda$. Each element $T_{i}$ is foliated by stable manifolds $W^{s}\left(x, T_{i}\right)=\{y \in$ $\left.T_{i}: f^{n} x, f^{n} y \in T_{i_{n}}, n \geq 0\right\}$. We can define an equivalence relation $x \sim y$ if $y \in W^{s}\left(x, T_{i}\right)$ and the equivalence classes are described by $\Sigma^{+}$. We can consider the one dimensional expanding map $S: X \rightarrow X$ where $X=\coprod_{i} T_{i} / \sim$ corresponds to identifying $\Lambda$ along the stable manifolds on each element of the Markov Partition. Since the lamination by stable manifolds is $C^{1+\alpha}$, for some $\alpha>0$, the corresponding map $S: X \rightarrow X$ is $C^{1+\alpha}$. As before we can show that the sets of points whose 
Birkhoff sums for $\Phi$ (restricted to some representative unstable leaf) have the same dimension if we assume the limit supremum is $\gamma$ or $\gamma_{0}$. Moreover, the dimension of the set of points in $\Lambda$ which are the union of those lying in a piece of stable leaf containing a point from the first set agrees with the dimension of the set of points in $\Lambda$ which are the union of those lying in a piece of stable leaf containing a point from the second set, using the natural Lipschitz local product structure. The result then follows.

\section{Final COMments}

We conclude with some comments on our earlier results.

(1) There is a simple analogue of Proposition 6.1 for the Central Limit Theorem. For any $t>0, \delta>0$ and $\epsilon>0$ there exists $n \geq 1$ and $\bar{\mu}$ a $\sigma^{n}$ invariant measure such that $\operatorname{dim} \bar{\mu}>\operatorname{dim} \mu-\delta$ and

$$
\lim _{n \rightarrow \infty} \bar{\mu}\left\{x \in \Sigma:-t \leq \frac{1}{\sqrt{n}} f^{n}(x) \leq t\right\} \leq \epsilon
$$

(2) If we consider the analogous problem with $\gamma<\gamma_{0}$ then our method of proof does not give a way to constructing Gibbs measures with less variance. In this case we only know the much weaker result that if $f(\alpha)>0$ then for any $\gamma>0$ we have $f(\alpha, \gamma)>0$.

(3) The assumption of the strong separation condition was merely one of convenience. In the case of iterated function systems the assumption of the strong separation condition can be weakened to the open set condition. This method can then be applied, for example, to repellers for conformal expanding maps to get similar results. This includes one dimension expanding Markov maps. The adjustment needed for this to work is that the Gibbs measures and potentials are defined on the associated shift space and then projected on to the iterated function system.

\section{REFERENCES}

1. R. Bowen, Equilibrium states and the ergodic theory of Anosov diffeomorphisms, Lecture Notes in Mathematics Vol. 470., Springer-Verlag, Berlin-New York, $1975 .$.

2. A. Broise, Transformations dilatantes de l'intervalle et théorèmes limites, Études spectrales d'opérateurs de transfert et applications, Astŕisque 238 (1996), 1-109.

3. R. Cawley and D. Mauldin, Multifractal decompositions of Moran fractals, Adv. Math. 92, 196-236.

4. M. Denker and W. Philipp, Approximation by Brownian motion for Gibbs measures and flows under a function, Ergodic Theory Dynam. Systems 4 (1984), 541-552.

5. K. Falconer, Fractal geometry. Mathematical foundations and applications, John Wiley and Sons, Inc., Hoboken, NJ, 2003.

6. K. Falconer, Techniques in Fractal geometry, John Wiley and Sons, Inc., Hoboken, NJ, 1997.

7. A.-H. Fan and J. Schmeling, On fast Birkhoff averaging, Math. Proc. Cambridge Philos. Soc. 135 (2003), 443-467.

8. Y. Guivarc'h and J. Hardy, Thormes limites pour une classe de chanes de Markov et applications aux diffomorphismes d'Anosov, Ann. Inst. H. Poincar Probab. Statist. 24 (1988), 73-98.

9. F. Ledrappier and A. Porzio, On the multifractal analysis of Bernoulli convolutions. II. Dimensions., J. Statist. Phys. 82 (1996), 397-420.

10. L. Olsen Slow and fast convergence to local dimensions of self-similar measures, Math. Nachr. 266 (2004), 68-80. 
11. L. Olsen A multifractal formalism, Advances in Mathematics 116 (1995), 82-196.

12. W. Parry and M. Pollicott, Zeta functions and the closed orbit structure of hyperbolic systems, Asterisque, 187-188.

13. Y. Pesin, Dimension theory in dynamical systems, Chicago Lectures in Mathematics, University of Chicago Press, Chicago, IL, 1997.

14. Y. Pesin and H. Weiss, The multifractal analysis of Gibbs measures: motivation, mathematical foundation, and examples, Chaos 7 (1997), 89-106.

15. Y. Pesin and H. Weiss, The multifractal analysis of Birkhoff averages and large deviations, Global analysis of dynamical systems, Fleschrift dedicated to Floris Takens for his 60th birthday, IOP, Bristol, pp. 419-431.

16. D. Rand, The singularity spectrum $f(\alpha)$ for cookie-cutters, Ergodic Theory Dynam. Systems 9 (1989), 527-541.

17. D. Ruelle, Thermodynamic Formalism, Wiley, New York, 1978.

18. J. Schmeling and H. Weiss, An overview of the dimension theory of dynamical systems, Smooth ergodic theory and its applications (Seattle, WA, 1999), Proc. Sympos. Pure Math., 69,, Amer. Math. Soc., Providence, RI, 2001, pp. 429-488,.

Mark Pollicott, Department of Mathematics, Warwick University, Coventry, CV4 7AL, UK

E-mail address: mpollic@maths.warwick.ac.uk

Thomas Jordan, Department of Mathematics, Warwick University, Coventry, CV4 $7 \mathrm{AL}, \mathrm{UK}$

E-mail address: tjordan@maths.warwick.ac.uk 